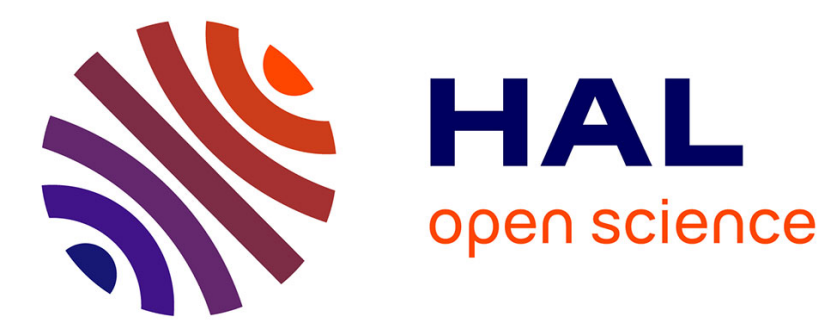

\title{
Outerplanar obstructions for matroid pathwidth
}

\author{
Athanassios Koutsonas, Dimitrios M. Thilikos, Koichi Yamazaki
}

\section{To cite this version:}

Athanassios Koutsonas, Dimitrios M. Thilikos, Koichi Yamazaki. Outerplanar obstructions for matroid pathwidth. Discrete Mathematics, 2014, 315, pp.95-101. 10.1016/j.disc.2013.10.007 . lirmm01225581

\section{HAL Id: lirmm-01225581 https://hal-lirmm.ccsd.cnrs.fr/lirmm-01225581}

Submitted on 6 Nov 2015

HAL is a multi-disciplinary open access archive for the deposit and dissemination of scientific research documents, whether they are published or not. The documents may come from teaching and research institutions in France or abroad, or from public or private research centers.
L'archive ouverte pluridisciplinaire HAL, est destinée au dépôt et à la diffusion de documents scientifiques de niveau recherche, publiés ou non, émanant des établissements d'enseignement et de recherche français ou étrangers, des laboratoires publics ou privés. 
Provided for non-commercial research and education use. Not for reproduction, distribution or commercial use.

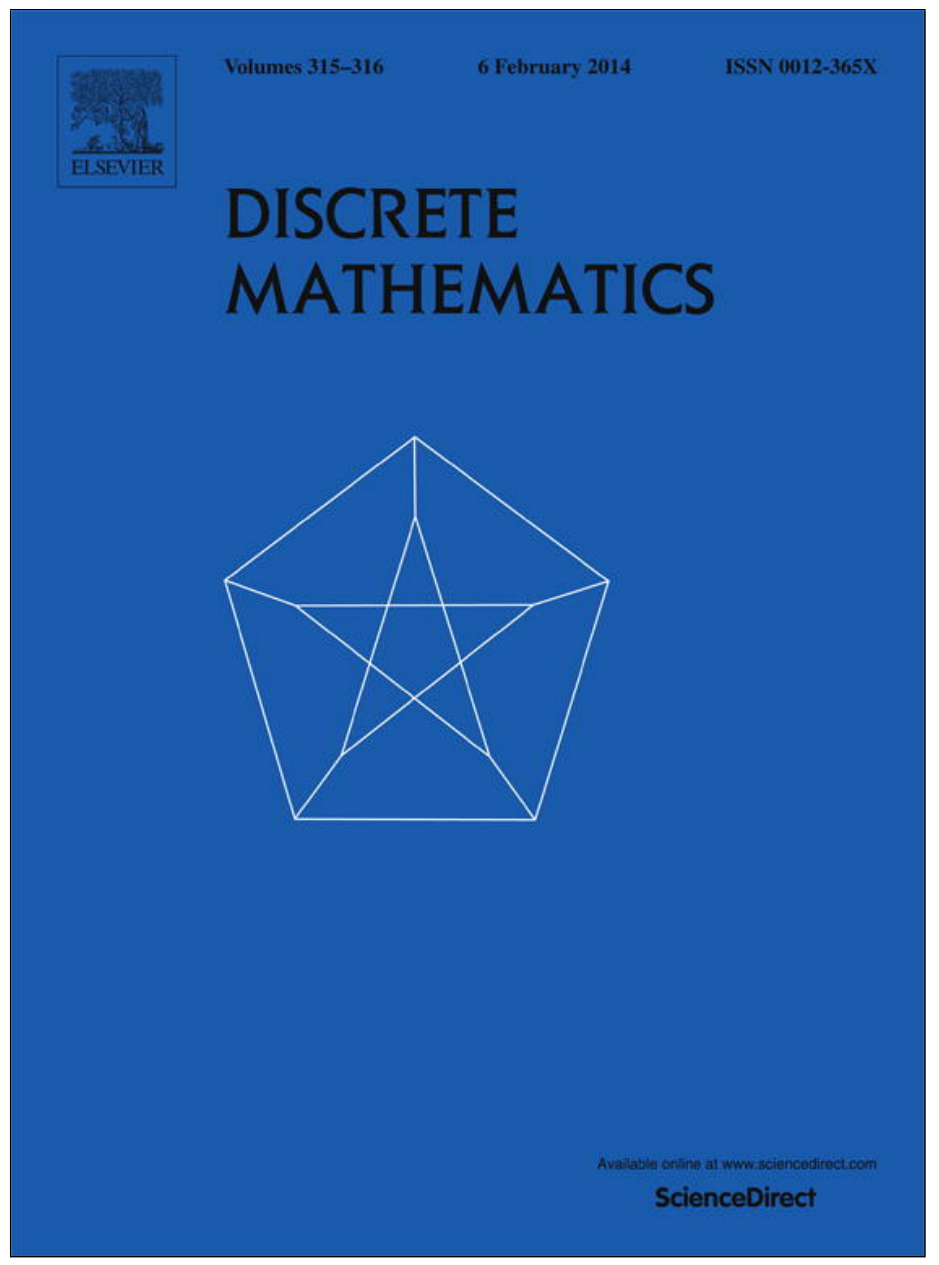

This article appeared in a journal published by Elsevier. The attached copy is furnished to the author for internal non-commercial research and education use, including for instruction at the authors institution and sharing with colleagues.

Other uses, including reproduction and distribution, or selling or licensing copies, or posting to personal, institutional or third party websites are prohibited.

In most cases authors are permitted to post their version of the article (e.g. in Word or Tex form) to their personal website or institutional repository. Authors requiring further information regarding Elsevier's archiving and manuscript policies are encouraged to visit:

http://www.elsevier.com/authorsrights 


\title{
Outerplanar obstructions for matroid pathwidth
}

\author{
Athanassios Koutsonas ${ }^{a}$, Dimitrios M. Thilikos ${ }^{\mathrm{a}, \mathrm{b}, *}$, Koichi Yamazaki $^{\mathrm{c}}$ \\ ${ }^{a}$ Department of Mathematics, National and Kapodistrian University of Athens, Panepistimioupolis, GR-15784 Athens, Greece \\ ${ }^{\mathrm{b}}$ AlGCo project-team, CNRS, LIRMM, France \\ ${ }^{\mathrm{c}}$ Department of Computer Science, Faculty of Engineering, Gunma University, Gunma, Japan
}

\section{A R T I C L E I N F O}

\section{Article history:}

Received 25 February 2012

Received in revised form 25 July 2013

Accepted 9 October 2013

Available online 7 November 2013

\section{Keywords:}

Matroids

Obstructions

Pathwidth

Outerplanar graphs

\begin{abstract}
A B S T R A C T
For each non-negative integer $k$, we provide all outerplanar obstructions for the class of graphs whose cycle matroid has pathwidth at most $k$. Our proof combines a decomposition lemma for proving lower bounds on matroid pathwidth and a relation between matroid pathwidth and linear width. Our results imply the existence of a linear algorithm that, given an outerplanar graph, outputs its matroid pathwidth.
\end{abstract}

(C) 2013 Elsevier B.V. All rights reserved.

\section{Introduction}

The notions of pathwidth and branchwidth are fundamental graph parameters that appear in many topics of discrete mathematics and algorithms. The counterpart of branchwidth on matroids has been introduced by Geelen and Whittle in [6] and was extensively studied in $[6,15,10,14,11,5]$. However, not much is known for the counterpart of pathwidth on matroids. The pathwidth of a matroid was defined by Geelen, Gerards, and Whittle in [7] (see also [9]) and was extensively studied in the work of Kashyap [13] in the context of trellis state-complexity of linear codes. Also, connected matroids of pathwidth at most 3 have been studied in $[8,1]$.

Given a class of matroids $\mathcal{M}$, we define its obstruction set obs( $\mathcal{M})$ as the set of all minor-minimal matroids not in $\mathcal{M}$ ( $\operatorname{similarly,~we~define~obs~}(\mathcal{G})$ for the case where $\mathcal{G}$ is a class of graphs). We define $\mathcal{P}_{k}$ as the class of all matroids of pathwidth at most $k$. In this paper, we study the set $\mathbf{o b s}\left(\mathcal{P}_{k}\right)$ and we characterize, for every $k$, all members of obs $\left(\mathcal{P}_{k}\right)$ that are cycle matroids of outerplanar graphs.

Following Kashyap [13], we define the matroid-pathwidth (in short: $\mu$-pathwidth) of a graph as the pathwidth of its cycle matroid. As observed in [13], the pathwidth and the $\mu$-pathwidth of a graph are different parameters, while the pathwidth can be computationally reduced to $\mu$-pathwidth.

In this paper, we show that several structural characteristics of the pathwidth of acyclic graphs are transferred to the $\mu$-pathwidth of outerplanar graphs. In particular, we define an operation, called fusion for "joining together" triples of matroids and we prove a structural result (Lemma 3) that provides a way to construct matroids of pathwidth at least $k+1$ from matroids of pathwidth at least $k$. Our result can be seen as the matroid-analogue of the operation defined in [19] (see also [20,17,3]) for the case of the pathwidth of graphs (see also [12] for related recent results on the parameter of linear rank-width).

\footnotetext{
* Corresponding author at: Department of Mathematics, National and Kapodistrian University of Athens, Panepistimioupolis, GR-15784 Athens, Greece. E-mail addresses: akoutson@math.uoa.gr (A. Koutsonas), sedthilk@thilikos.info (D.M. Thilikos), koichi@cs.gunma-u.ac.jp (K. Yamazaki).
} 
Using our structural lemma, we prove the existence of a bijection between acyclic obstructions for linear-width (a parameter very similar to the pathwidth for graphs) and the outerplanar obstructions of $\mu$-pathwidth. This gives a precise characterization of all members of $\mathbf{o b s}\left(\mathcal{P}_{k}\right)$ that are cycle matroids of outerplanar graphs. A byproduct of our results is that the $\mu$-pathwidth of outerplanar graphs can be computed in linear time.

\section{Definitions and preliminaries}

Given a graph $G$, we denote by $V(G)$ its vertex set and as $E(G)$ its edge set. We consider graphs that may have loops or multiple edges. If a graph has no multiple edges or loops we call it simple. For any set of vertices $S \subseteq V(G)$, we denote by $G[S]$ the subgraph of $G$ induced by the vertices in $S$. Accordingly, for a set of edges $F \subseteq E(G)$, we define $G[F]=(V(F), F)$ where $V(F)=\bigcup_{e \in F}$. In addition, we define by $\bar{F}=E(G)-F$.

We use the term plane graph for a planar graph along with an embedding of it in the sphere $\mathbb{S}_{0}$ without crossings. To simplify notations, we do not distinguish between a vertex of the graph and the point of $\mathbb{S}_{0}$ used in the drawing to represent the vertex or between an edge and the open line segment representing it. We denote by $F(G)$, the set of faces of this embedding, i.e. the connected components of $\mathbb{S}_{0} \backslash G$, (that are open sets of $\mathbb{S}_{0}$ ). A planar graph $G$ is outerplanar if it has an embedding on the sphere $\mathbb{S}_{0}$ such that all its vertices lie on the boundary of a single face, called the outer face.

Given two graphs $H$ and $G$, we write $H \preceq G$ and call $H$ a minor of $G$, if $H$ can be obtained by a subgraph of $G$ after a series of edge contractions (the contraction of an edge $e=\{x, y\}$ in a graph $G$ is the operation of replacing $x$ and $y$ by a new vertex $x_{e}$ that is made adjacent with all the neighbors of $x$ and $y$ in $G$ that are different from $x$ and $y$ ). We also use the notation $G^{*}$ to denote an embedding of the dual graph of $G$.

Matroids and cycle matroids of graphs. Given a matroid $\mathcal{M}$, we use the notations $E(\mathcal{M})$ and $\ell(\mathcal{M})$ for its elements and the collection of its independent sets, respectively. Hence $\mathcal{M}=\{E(\mathcal{M}), \ell(\mathcal{M})\}$. We also call $E(\mathcal{M})$ the ground set of $\mathcal{M}$. Moreover, following the notation of [16], we denote by $\mathcal{B}(\mathcal{M})$ the collection of the maximal independent sets, the bases of $\mathcal{M}$ and $\mathcal{C}(\mathcal{M})$ the collection of the minimal dependent sets, the circuits of $\mathcal{M}$. It is known (see e.g., [16]) that any of the collections $\ell(\mathcal{M}), \mathcal{B}(\mathcal{M})$ or $\mathcal{C}(\mathcal{M})$ suffices to describe a matroid $\mathcal{M}$ on an element set $E(\mathcal{M})$.

The cycle matroid of a graph $G$, denoted as $\mathcal{M}(G)$, has $E(G)$ as ground set, while its independent sets are the sub-forests of $G$. On the other hand, a matroid that is isomorphic to a cycle matroid of a graph is called graphic.

Given a matroid $\mathcal{M}$, the matroid whose ground set is $E(\mathcal{M})$ and whose collection of bases is $\{E(\mathcal{M})-B: B \in \mathscr{B}(\mathcal{M})\}$ is called the dual of the matroid $\mathcal{M}$ and is denoted by $\mathcal{M}^{*}$.

Let $\mathcal{M}$ be a matroid and $X, Y \subseteq E(\mathcal{M})$ subsets of its ground set. The matroid $\mathcal{M} \backslash X=\{E(\mathcal{M})-X, I \subseteq(E(\mathcal{M})-X): I \in$ $\ell(\mathcal{M})\}$ and the matroid $\mathcal{M} / Y=\left[\mathcal{M}^{*} \backslash Y\right]^{*}$ are the deletion of $X$ and the contraction of $Y$ from the matroid $\mathcal{M}$, respectively. If $X=\{e\}$ then we simply write $\mathcal{M} \backslash e$ instead of $\mathcal{M} \backslash\{e\}$-likewise for the contraction of a single element. A matroid $\mathcal{K}$ is a minor of a matroid $\mathcal{M}$ if $K=\mathcal{M} \backslash X / Y$ for some $X, Y \subseteq E(\mathcal{M})$.

Using the definition of Oxley [16] the matroid connectivity function $\lambda: 2^{E(\mathcal{M})} \rightarrow \mathbb{N}$ is defined as follows:

$$
\lambda_{\mathcal{M}}(X)=r_{\mathcal{M}}(X)+r_{\mathcal{M}}(\bar{X})-r_{\mathcal{M}}(E(\mathcal{M}))
$$

where $\bar{X}=E(\mathcal{M})-X$ and $r$ is the rank function of $\mathcal{M}$, i.e. $r_{\mathcal{M}}(X)$ is the maximum cardinality of an independent set of $X$ in $\mathcal{M}$.

A matroid that for every pair of distinct elements of its ground set has a circuit containing both of them is called connected. This implies that the cycle matroid of a 2-connected graph is connected. It is also easy to check that if a matroid $\mathcal{M}$ is connected and $F \subseteq E(\mathcal{M})$, then $\lambda_{\mathcal{M}}(F)=0$ only if $F=E(\mathcal{M})$ or $F=\emptyset$.

Another useful property of connectivity is the following.

Fact 1 ([22]). Let e be an element of a connected matroid $\mathcal{M}$. Then at least one of $\mathcal{M} / e$ and $\mathcal{M} \backslash$ e is connected.

Given two matroids $\mathcal{M}_{1}$ and $\mathcal{M}_{2}$ on disjoint ground sets, we denote by $\mathcal{M}_{1} \oplus \mathcal{M}_{2}$ their direct sum, i.e. the matroid $\left(E\left(\mathcal{M}_{1}\right) \cup E\left(\mathcal{M}_{2}\right), \ell\left(\mathcal{M}_{1}\right) \cup \ell\left(\mathcal{M}_{2}\right)\right)$.

We continue with the definition of two dual operations on matroids which will play an important role in the proofs of the next section. If an element is not contained in a base of a matroid, then it is called loop, while if it is in every base is called coloop. Then, given two matroids $\mathcal{M}_{1}$ and $\mathcal{M}_{2}$ with $E\left(\mathcal{M}_{1}\right) \cap E\left(\mathcal{M}_{2}\right)=\{e\}$ where $e$ is neither a loop nor a coloop in these matroids, the series connection of $\mathcal{M}_{1}$ and $\mathcal{M}_{2}$, denoted by $S\left(\mathcal{M}_{1}, \mathcal{M}_{2}\right)$, is the matroid with element $\operatorname{set} E\left(\mathcal{M}_{1} \backslash e\right) \cup E\left(\mathcal{M}_{2} \backslash e\right) \cup e^{\prime}$, where $e^{\prime}$ is an element not in $E\left(\mathcal{M}_{1}\right)$ or $E\left(\mathcal{M}_{2}\right)$ and whose collection of circuits is:

$$
\mathcal{C}_{S}=\mathcal{C}\left(\mathcal{M}_{1} \backslash e\right) \cup \mathcal{C}\left(\mathcal{M}_{2} \backslash e\right) \cup\left\{\left(C_{1}-e\right) \cup\left(C_{2}-e\right) \cup e^{\prime}: e \in C_{i} \in \mathcal{C}\left(\mathcal{M}_{i}\right) \text { for } i=1,2\right\} .
$$

Subsequently, the parallel connection of $\mathcal{M}_{1}$ and $\mathcal{M}_{2}$ denoted by $P\left(\mathcal{M}_{1}, \mathcal{M}_{2}\right)$ is the matroid $\left[S\left(\mathcal{M}_{1}^{*}, \mathcal{M}_{2}^{*}\right)\right]^{*}$. Properties of series and parallel connections include many attractive features. As example the classes of graphic matroids and connected matroids are closed under these operations-for a further study see also [16]. We will need the following property from [2].

Fact 2. Let $\mathcal{N}_{1}, \mathcal{N}_{2}$ be two matroids where $E\left(\mathcal{N}_{1}\right) \cap E\left(\mathcal{N}_{2}\right)=\{e\}$. Then, $P\left(\mathcal{N}_{1}, \mathcal{N}_{2}\right) / e=\left(\mathcal{N}_{1} / e\right) \oplus\left(\mathcal{N}_{2} / e\right)$. 
Matroid pathwidth. Given a layout (an ordering) $L=\left(e_{1}, \ldots, e_{m}\right)$ of $E(\mathcal{M})$, we define

$$
\mu \text {-width }{ }_{\mathcal{M}}(L)=\max \left\{\lambda_{\mathcal{M}}\left(\left\{e_{1}, \ldots, e_{i}\right\}\right) \mid 1 \leq i \leq m-1\right\} .
$$

According to the definition of [7] the pathwidth of a matroid $\mathcal{M}$, denoted by $\mathbf{p w}(\mathcal{M})$, is the minimum $k$ for which there exists a layout $L=\left(e_{1}, \ldots, e_{m}\right)$ of its elements such that $\mu$-width $\mathcal{M}(L) \leq k$.

The matroid pathwidth of a graph $G$ (or simply $\mu$-pathwidth) is defined as the pathwidth of its cycle matroid and it is denoted as $\mu-\mathbf{p w}(G)$, in other words:

$$
\mu-\mathbf{p w}(G)=\mathbf{p w}(\mathcal{M}(G)) .
$$

We will need the following simple lemma.

Lemma 1. The $\mu$-pathwidth of a graph is equal to the maximum $\mu$-pathwidth of all its biconnected components.

Proof. Let $\left\{C_{1}, \ldots, C_{r}\right\}$ be the biconnected components of a graph $G$ and $E_{1}, \ldots, E_{r}$ the corresponding edge sets. Then, for $i=1, \ldots, r$ it holds that $r_{\mathcal{M}}\left(E_{i}\right)+r_{\mathcal{M}}\left(\overline{E_{i}}\right)=r(\mathcal{M})$, where by $\mathcal{M}$ is denoted the cycle matroid of $G$. This implies that the value of the connectivity function of a set of edges belonging to the same component, is irrelevant from the rest of the biconnected components. This implies that if $\mu$-width $\left(L_{i}\right) \leq k$ for $i=1, \ldots, r$, where $L_{i}$ is a layout of $E_{i}$, then for the concatenation $L$ of all these layouts it also holds $\mu$-width $(L) \leq k$.

\section{A decomposition of matroids}

Let $\mathcal{M}_{1}, \mathcal{M}_{2}, \mathcal{M}_{3}$ be connected matroids on disjoint edge sets and for each of them pick an element $e_{i} \in E\left(\mathcal{M}_{i}\right), i=1,2,3$. For the uniform matroid $U_{1,3}$ with elements $\left\{e_{1}, e_{2}, e_{3}\right\}$ we can associate one common element with each of the three matroids: $E\left(\mathcal{U}_{1,3}\right) \cap E\left(\mathcal{M}_{i}\right)=\left\{e_{i}\right\}$. We call fusion of $\mathcal{M}_{1}, \mathcal{M}_{2}, \mathcal{M}_{3}$ on the elements $\left\{e_{1}, e_{2}, e_{3}\right\}$ the matroid

$$
\mathcal{M}=S\left(S\left(S\left(\mathcal{U}_{1,3}, \mathcal{M}_{1}\right), \mathcal{M}_{2}\right), \mathcal{M}_{3}\right)
$$

and we denote it by fusion $\left(\mathcal{M}_{1}, \mathcal{M}_{2}, \mathcal{M}_{3}, e_{1}, e_{2}, e_{3}\right)$. It is easy to check that this matroid is also connected. The elements $e_{1}, e_{2}, e_{3}$ on which the fusion takes place will be referred to as bridge elements.

The series connections in the definition of $\mathcal{M}$ involve the three elements of $u_{1,3}$ which implies that the order in which the connections are performed is irrelevant. Consider now the matroid $S\left(S\left(U_{1,3}, \mathcal{M}_{1}\right), \mathcal{M}_{2}\right)$ one step before the composition of the final matroid $\mathcal{M}$. Let $\mathcal{M}^{(1)}$ and $\mathcal{M}^{(2)}$ be matroids isomorphic to $U_{1,2}$ where $E\left(\mathcal{M}^{(1)}\right)=\left\{e_{1}, e_{3}\right\}$ and $E\left(\mathcal{M}^{(2)}\right)=\left\{e_{2}, e_{3}\right\}$. Keeping in mind that $U_{1,3}$ is isomorphic to $P\left(\mathcal{M}^{(1)}, \mathcal{M}^{(2)}\right)$ we can observe the following.

Observation 1. The matroid $P\left(S\left(\mathcal{M}^{(1)}, \mathcal{M}_{1}\right), S\left(\mathcal{M}^{(2)}, \mathcal{M}_{2}\right)\right)$ is isomorphic to the matroid $S\left(S\left(U_{1,3}, \mathcal{M}_{1}\right), \mathcal{M}_{2}\right)$.

In order to estimate the pathwidth of the fusion of three given matroids we need the following lemma about the connectivity function of a matroid formed by a series connection.

Lemma 2. Let $\mathcal{M}_{1}, \mathcal{M}_{2}$ be connected matroids with $E\left(\mathcal{M}_{1}\right) \cap E\left(\mathcal{M}_{2}\right)=\{e\}$ and $\mathcal{M}=S\left(\mathcal{M}_{1}, \mathcal{M}_{2}\right)$. For any two sets $F_{1}, F_{2}$ where $F_{1} \subseteq E\left(\mathcal{M}_{1}\right)$ and $F_{2} \subseteq E\left(\mathcal{M}_{2} \backslash e\right)$ it holds that

$$
\lambda_{M}\left(F_{1} \cup F_{2}\right) \geq \lambda_{\mathcal{M}_{1}}\left(F_{1}\right)+\lambda_{\mathcal{M}_{2} \backslash e}\left(F_{2}\right) .
$$

Proof. Let $\mathcal{M}_{i}^{-}=\mathcal{M}_{i} \backslash e, i=1$, 2. Since $\mathcal{M}_{1}, \mathcal{M}_{2}$ are both connected matroids it follows that their series connection $\mathcal{M}$ is also connected. From the connectivity of $\mathcal{M}_{i}$, we obtain that $r\left(\mathcal{M}_{i}\right)=r\left(\mathcal{M}_{i}^{-}\right), i=1$, 2. Also, from the connectivity of $\mathcal{M}$ it follows that $r(\mathcal{M})=r(\mathcal{M} \backslash e)$. Notice that $\mathcal{M}=S\left(\mathcal{M}_{1}, \mathcal{M}_{2}\right)$ implies that $\mathcal{M} \backslash e=\mathcal{M}_{1}^{-} \oplus \mathcal{M}_{2}^{-}$, which, in turn, implies that $r(\mathcal{M})=r\left(\mathcal{M}_{1}\right)+r\left(\mathcal{M}_{2}^{-}\right)$. From the definition of the connectivity function $\lambda$ and the last equality, it is enough to prove that for any two subsets $F_{1} \subseteq E\left(\mathcal{M}_{1}\right), F_{2} \subseteq E\left(\mathcal{M}_{2}^{-}\right)$,

$$
\begin{aligned}
& r_{M}\left(F_{1} \cup F_{2}\right) \geq r_{\mathcal{M}_{1}}\left(F_{1}\right)+r_{\mathcal{M}_{2}^{-}}\left(F_{2}\right) \\
& r_{M}\left(\overline{F_{1} \cup F_{2}}\right) \geq r_{\mathcal{M}_{1}}\left(\overline{F_{1}}\right)+r_{\mathcal{M}_{2}^{-}}\left(\overline{F_{2}}\right) .
\end{aligned}
$$

Towards a contradiction, suppose that $r_{M}\left(F_{1} \cup F_{2}\right)<r_{\mathcal{M}_{1}}\left(F_{1}\right)+r_{\mathcal{M}_{2}^{-}}\left(F_{2}\right)$ and let $B_{1}, B_{2}$ be bases of $F_{1}, F_{2}$ respectively. Then $B_{1} \cup B_{2}$ should contain a circuit $C$ in $\mathcal{M}$. Moreover, since $B_{1} \cap B_{2}=\emptyset$ the circuit $C$ has elements from both $B_{1}, B_{2}-$ as otherwise it would contradict their choice as bases. Contracting the elements of $\mathcal{M}_{2}^{-}$in $\mathcal{M}$ will force the existence of a circuit $C_{1} \subseteq C \cap E\left(\mathcal{M}_{1}\right)=B_{1}$ in $M / E\left(\mathcal{M}_{2}^{-}\right)=\mathcal{M}_{1}$ which contradicts that $B_{1}$ is a base in $\mathcal{M}_{1}$ and completes the proof of (1). Then (2) easily follows from (1) by the symmetry of the connectivity function $\lambda$ and the fact that $\bar{F}_{1} \cup \bar{F}_{2}=\overline{F_{1} \cup F_{2}}$ (recall that $\left.F_{1} \cap F_{2}=\emptyset\right)$.

We are now ready to prove the main structural result of this paper. 

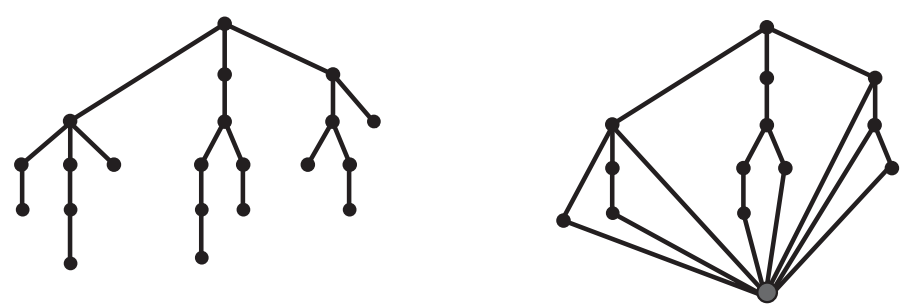

Fig. 1. An example of the application of the function $\phi$.

Lemma 3. Let $\mathcal{M}$ be a matroid that is obtained by the fusion of three connected matroids $\mathcal{M}_{a}, \mathcal{M}_{b}, \mathcal{M}_{c}$. Then it holds that

$$
\mathbf{p w}(\mathcal{M}) \geq \min \left\{\mathbf{p w}\left(\mathcal{M}_{a}\right), \mathbf{p w}\left(\mathcal{M}_{b}\right), \mathbf{p w}\left(\mathcal{M}_{c}\right)\right\}+1 .
$$

Proof. We will prove that, for any layout $L=\left(e_{1}, \ldots, e_{m}\right)$ of the element set $E(\mathcal{M})$ of $\mathcal{M}$, there is an $q \in\{1, \ldots, m-1\}$ such that $\lambda_{\mathcal{M}}\left(\left\{e_{1}, \ldots, e_{q}\right\}\right) \geq k+1$ where $k=\min \left\{\mathbf{p w}\left(\mathcal{M}_{a}\right), \mathbf{p w}\left(\mathcal{M}_{b}\right), \operatorname{pw}\left(\mathcal{M}_{c}\right)\right\}$.

We denote by $e_{a}, e_{b}, e_{c} \in E(\mathcal{M})$ the bridge elements of the fusion associated with the matroids $\mathcal{M}_{a}, \mathcal{M}_{b}, \mathcal{M}_{c}$ respectively and, for simplicity, we use the notation $E_{a}=E\left(\mathcal{M}_{a}\right), E_{b}=E\left(\mathcal{M}_{b}\right)$ and $E_{c}=E\left(\mathcal{M}_{c}\right)$. Assume w.l.o.g. that $e_{1} \in E_{a}$. Likewise, we assume that the last, in $L$, element $e_{\ell}$ of $E(\mathcal{M}) \backslash E_{a}$ belongs in $E_{c}$. Note then that all edges of $E_{b}$ appear in $L$ after $e_{1} \in E_{a}$ and before $e_{\ell} \in E_{c}$.

By the definition of fusion follows that $\mathcal{M} /\left(E_{a} \cup E_{c}\right)=\mathcal{M}_{b}$. Consider the matroid $\mathcal{M}^{\prime}=\mathcal{M} /\left(E_{b} \backslash e_{b}\right)$ and observe that $\mathcal{M}^{\prime}=S\left(S\left(U_{1,3}, \mathcal{M}_{a}\right), \mathcal{M}_{c}\right)$. Let $L_{b}=\left(e_{p_{1}}, \ldots, e_{p_{s}}\right)$ be the restriction of $L$ in $E\left(\mathcal{M}_{b}\right)$. As $\mathbf{p w}\left(\mathcal{M}_{b}\right) \geq k$, there is an $h \in\{1, \ldots, s-1\}$ such that $\lambda_{\mathcal{M}_{b}}\left(F_{b}\right) \geq k$, where $F_{b}=\left\{e_{p_{1}}, \ldots, e_{p_{h}}\right\} \subseteq E_{b}$. Let $F^{\prime}=\left\{e_{j} \in L \mid e_{j} \notin E_{b}\right.$ and $\left.j<p_{h}\right\}$.

Observe now that $F^{\prime} \subseteq E_{a} \cup E_{c}$ and $\left\{e_{1}, \ldots, e_{p_{h}}\right\}=F_{b} \cup F^{\prime}$. Since $E\left(\mathcal{M}^{\prime} \backslash e_{b}\right)=E_{a} \cup E_{c}$, it also holds that $F^{\prime} \subseteq E\left(\mathcal{M}^{\prime} \backslash e_{b}\right)$. From Observation $1, M^{\prime}$ can be seen as a parallel connection of two matroids on the element $e_{b}$. This, together with Fact 2 implies that $\mathcal{M}^{\prime} / e_{b}$ is not connected. By Fact 1 , it follows that the matroid $\mathcal{M}^{\prime} \backslash e_{b}$ is connected.

By the connectivity of $\mathcal{M}^{\prime} \backslash e_{b}$ and the fact that $e_{1} \in F^{\prime}, e_{\ell} \notin F^{\prime}$ we obtain that $\lambda_{\mathcal{M}^{\prime} \backslash e_{b}}\left(F^{\prime}\right) \geq 1$. Observe finally that $\mathcal{M}^{\prime} \cap \mathcal{M}_{b}=\left\{e_{b}\right\}$ and $\mathcal{M}=S\left(\mathcal{M}^{\prime}, \mathcal{M}_{b}\right)$. Applying Lemma 2 implies that $\lambda_{M}\left(F_{b} \cup F^{\prime}\right) \geq k+1$. As $\left\{e_{1}, \ldots, e_{p_{h}}\right\}=F_{b} \cup F^{\prime}$, we can choose $q=p_{h}$.

\section{4. $\mu$-pathwidth and linear width}

Let $\mathcal{T}$ be the set of all trees. We define the function $\phi$ that maps trees to graphs such that for every $T \in \mathcal{T}, \phi(T)$ is the graph obtained if we identify all the leaves of $T$ to a single vertex (see Fig. 1 for an example). We denote the new vertex as the join-vertex of $\phi(T)$.

Observe that if $G$ is a 2-connected outerplanar graph, then its dual $H$ belongs to the class $\phi(\mathcal{T})$, where its join-vertex corresponds to the outer face of $G$.

Let $G$ be a graph. For any set of edges $F \subseteq E(G)$ we denote by $\partial_{G}(F)$ the set of vertices of the graph that are incident with an edge in $F$ and also with an edge in $E(G) \backslash F$. The boundary function $\delta_{G}(F)$ of the graph $G$ is defined as $\delta_{G}(F)=\left|\partial_{G}(F)\right|$. We define the linear width of a graph $G$ as the minimum integer $k$ for which there exists a layout $L=\left(e_{1}, \ldots, e_{m}\right)$ of the edge set $E(G)$, such that $\max \left\{\delta_{G}\left(\left\{e_{1}, \ldots, e_{i}\right\}\right) \mid 1 \leq i \leq m-1\right\} \leq k$ and we write $\operatorname{lw}(G) \leq k$.

Given a graph $G$ and an edge set $F \subseteq E(\bar{G})$ as before, we denote by $\sigma_{G}(F)$ the number of the connected components of $G[F]$. We will need the following well known fact (see, e.g. $[16,15,6])$.

Fact 3. Let $G$ be a connected graph and $F \subseteq E(G)$. Then $\lambda_{\mathcal{M}(G)}(F)=\delta_{G}(F)-\sigma_{G}(F)-\sigma_{G}(\bar{F})+1$.

Using this fact we can prove the following relation between the linear width of a tree $T$ and the $\mu$-pathwidth of $\phi(T)$.

Lemma 4. For every tree $T$, it holds that $\mu-\mathbf{p w}(\phi(T)) \leq \mathbf{l} \mathbf{w}(T)$.

Proof. Let $F$ be an edge set in $E(T)$, where $F \neq \varnothing$ and $F \neq E(T)$. By the definition of the function $\phi$, the tree $T$ and the graph $H=\phi(T)$ share a common edge set. Note that only non-leaf vertices of $T$ contribute to $\delta_{T}(F)$ and observe that the corresponding vertices of $H$, with the possible addition of the vertex $v$, into which the leaves of $T$ merged, are the ones that contribute to $\delta_{H}(F)$; i.e. $\delta_{H}(F) \leq \delta_{T}(F)+1$. From Fact 3, we obtain that $\lambda_{\mathcal{M}(H)}(F)=\delta_{H}(F)-\sigma_{H}(F)-\sigma_{H}(\bar{F})+1 \leq \delta_{H}(F)-1 \leq$ $\delta_{T}(F)$. It is straightforward now to conclude that given any sequence of $E(T)$ that results to $\operatorname{lw}(T) \leq \ell$, the same sequence results to $\mu-\mathbf{p w}(H) \leq \ell$.

We recursively define the parameterized family of trees $\mathcal{T}_{k}$, for any non-negative integer $k$, as follows:

- Let $\mathcal{T}_{0}$ contain the tree obtained by the 1 -subdivision of $K_{2}$.

- For $k \geq 1, \mathcal{T}_{k}$ contains any tree that can be obtained by the following procedure: Take three (not necessarily distinct) members of $\mathcal{T}_{k-1}$, add a new vertex and connect it with some non-leaf vertex in each of these three trees. As long as a leaf in the resulting graph has a neighbor of degree 3 , delete this leaf. 

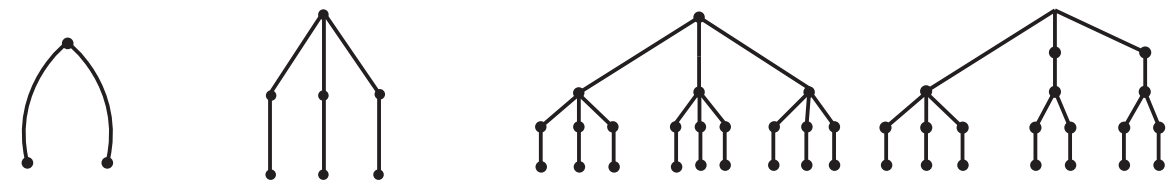

Fig. 2. The classes $\mathcal{T}_{0}, \mathcal{T}_{1}$, and part of $\mathcal{T}_{2}$.
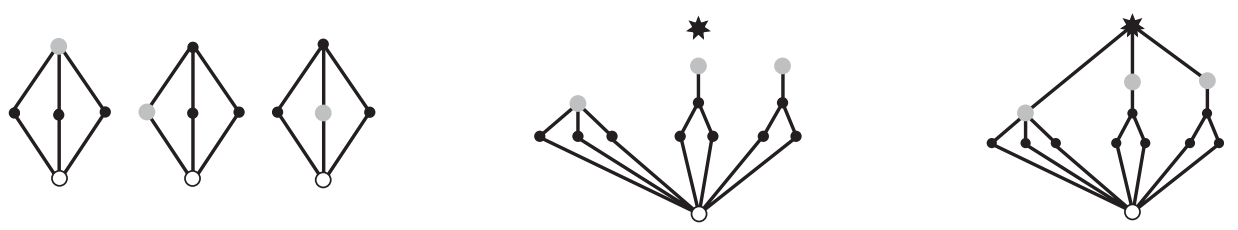

Fig. 3. The $u$-fusion operation.
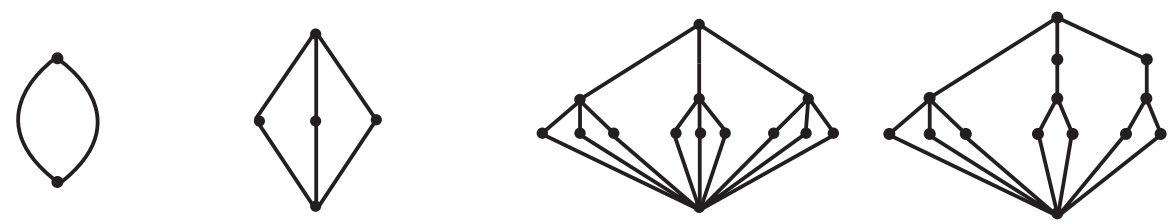

Fig. 4. The sets $\mathscr{H}_{0}, \mathscr{H}_{1}$, and part of $\mathscr{H}_{2}$.

For an example of the above construction, see Fig. 2 (only two of the four members of the class $\mathcal{T}_{2}$ are depicted in Fig. 2).

We denote by $\mathcal{L}_{k}$ the class of graphs with linear-width at most $k$. Linear-width is a parameter defined in [21] and studied in $[4,20]$. While it differs by at most one from the more known parameter of pathwidth, it is more easy to study in our context because of the strong similarity of its definition to the one of $\mu$-pathwidth.

The acyclic obstructions for $\mathcal{L}_{k}$ are determined by the following result (see Theorem 29 in [20]).

Proposition 1. For every non-negative integer $k, \boldsymbol{o b s}\left(\mathscr{L}_{k}\right) \cap \mathcal{T}=\mathcal{T}_{k}$.

\section{Obstructions for $\mu$-pathwidth}

Let now $G_{1}, G_{2}, G_{3}$ be three disjoint 2-connected graphs and $v_{i}, u_{i} \in V\left(G_{i}\right)$ a pair of distinct vertices for $i=\{1,2,3\}$. We call $u$-fusion of the graphs $G_{1}, G_{2}, G_{3}$ on the given pairs of vertices the graph $G$ constructed as follows:

(a) For $i=\{1,2,3\}$ if the vertices $v_{i}, u_{i}$ are adjacent in $G_{i}$, then delete the edge $\left\{v_{i}, u_{i}\right\}$ in $G_{i}$ (in case $\left\{v_{i}, u_{i}\right\}$ is a multiple edge, delete only one of its copies)

(b) Identify vertices $v_{1}, v_{2}, v_{3}$ to a single vertex $v$, take a new vertex $u$ not in $G_{1}, G_{2}$ or $G_{3}$, and add the edges $\left\{u_{1}, u\right\},\left\{u_{2}, u\right\}$ and $\left\{u_{3}, u\right\}$ (see Fig. 3 ).

Notice that $G$ is 2 -connected by construction. We will call the vertices $v$, $u$ base vertex and top vertex of the resulting graph respectively and the three edges incident to $u$ bridge edges in $G$.

Lemma 5. Let $G$ be a u-fusion of three disjoint 2-connected graphs $G_{1}, G_{2}$ and $G_{3}$. Then $\mu-\mathbf{p w}(G) \geq \min \left\{\mu-\mathbf{p w}\left(G_{1}\right)\right.$, $\mu$-pw $\left(G_{2}\right)$, $\left.\mu-\mathbf{p w}\left(G_{3}\right)\right\}+1$.

Proof. For $i=1,2$, 3 let us denote by $v_{i}, u_{i} \in V\left(G_{i}\right)$ the pair of vertices involved in the $u$-fusion of the three graphs. Consider for each graph $G_{i}$ the graph $G_{i}^{+}$which has the same vertex set as $G_{i}$ and edge set $E\left(G_{i}^{+}\right)=E\left(G_{i}\right)$ if $e_{i}=\left\{v_{i}, u_{i}\right\} \in E\left(G_{i}\right)$ or else $E\left(G_{i}^{+}\right)=E\left(G_{i}\right) \cup e_{i}$. Clearly $\mu-\mathbf{p w}\left(G_{i}^{+}\right) \geq \mu$-pw $\left(G_{i}\right)$ for $i=1,2,3$.

Recall that, by its definition, a matroid formed as a fusion of three graphic matroids is itself graphic as the class of graphic matroids is closed under series connection. By construction, the cycle matroid of $G$ is isomorphic to the matroid obtained by the fusion of the cycle matroids of the graphs $G_{1}^{+}, G_{2}^{+}, G_{3}^{+}$on the elements $e_{1}, e_{2}, e_{3}$. Then, the application of Lemma 3 yields that $\mu-\mathbf{p w}(G) \geq \min \left\{\mu-\mathbf{p w}\left(G_{1}\right), \mu-\mathbf{p w}\left(G_{2}\right), \mu-\mathbf{p w}\left(G_{3}\right)\right\}+1$.

For each non-negative integer $k$, we define $\mathscr{H}_{k}=\phi\left(\mathcal{T}_{k}\right)$, i.e. a graph $H$ belongs to the class $\mathscr{H}_{k}$, if and only if $H=\phi(T)$ for some $T \in \mathcal{T}_{k}$ (see also Fig. 4).

Under a closer look it becomes apparent, that the $u$-fusion can parallel the mechanism which generates the family $\mathcal{T}_{k}$, in such a way, that the family $\mathscr{H}_{k}$ can be similarly generated by the $u$-fusion, starting with the graph $C_{2}-$ the only member of the class $\mathcal{H}_{0}$. This fact is demonstrated by the following lemma. (We stress though, that an arbitrary application of the $u$-fusion on graphs from the class $\mathscr{H}_{k-1}$ will not necessarily generate a graph in $\mathscr{H}_{k}$.)

Lemma 6. Any graph in $\mathscr{H}_{k}$, where $k \geq 1$, is a $u$-fusion of three graphs of the class $\mathscr{H}_{k-1}$. 

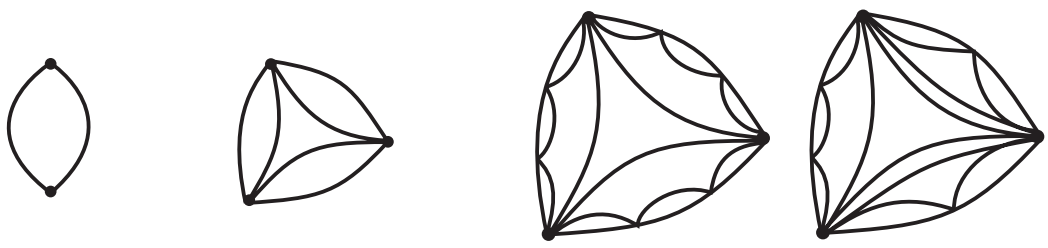

Fig. 5. The sets $\mathscr{H}_{0}^{*}, \mathscr{H}_{1}^{*}$ and part of $\mathscr{H}_{2}^{*}$.

Proof. Let $H$ be a graph in $\mathscr{H}_{k}$. Then, there exists a tree $T$ in $\mathcal{T}_{k}$, such that $H=\phi(T)$. Recall that $T$ is obtained from three trees of $\mathcal{T}_{k-1}$, say $T_{1}, T_{2}$ and $T_{3}$, by the recursive procedure described in the definition of the family of trees. We denote as $t$ the new vertex added during the construction of $T$, as $h$ the corresponding vertex of $H$ and as $t_{i}$ for $i \in\{1,2,3\}$ the vertex of $T_{i}$ joined to $t$.

Let now $H_{i}=\phi\left(T_{i}\right)$ and $v_{i}, u_{i}$ be a pair of vertices of $H_{i}$, where $v_{i}$ is the join-vertex of $H_{i}$ and $u_{i}$ is corresponding to the vertex $t_{i}$ of $T_{i}$. By definition, the vertex $t_{i}$ cannot be a leaf in $T_{i}$ and thus vertices $v_{i}, u_{i}$ are distinct. Then, let $U$ be the $u$-fusion of $H_{1}, H_{2}$ and $H_{3}$ on the given pairs of vertices and denote as $u$ and $v$ the top and the base vertex of $U$, respectively. We will show that $H$ is isomorphic to the graph $U$.

By associating the vertex $h$ with $u$ and $j$, the join-vertex of $H$, with $v$, we effectively define a bijection between $V(H)$ and $V(U)$, since all other vertices of both graphs correspond to the non-leaf vertices of $T_{1}, T_{2}$ and $T_{3}$. The choice of the vertices $u_{i}$, implies that the neighbors of $h$ in $H$ and $u$ in $U$ match under this bijection. Consider now that $t_{i}$ is adjacent to a leaf of $T_{i}$ (for some $i \in\{1,2,3\})$. Then, $u_{i}$ is adjacent to $v_{i}$ in $H_{i}$ and hence, this edge is deleted during the $u$-fusion, just as the neighboring leaf of $t_{i}$ is deleted during the formation of $T$. It follows that the neighbors of $j$ in $H$ and $v$ in $U$ are also matched.

We use Lemmata 5 and 6 to prove the following lemma.

Lemma 7. Let $H$ be a graph in $\mathscr{H}_{k}$ for some non-negative integer $k$. Then $H$ is an obstruction for $\mu$-pathwidth less than or equal to $k$.

Proof. Following the standard course for a proof of an obstruction set let us first attend to the value of the $\mu$-pathwidth of a given graph in $\mathscr{H}_{k}$. Notice that $\mu$-pw $\left(C_{2}\right)=1$ and that, for any $k \geq 1$, every graph in $\mathscr{H}_{k}$ is 2-connected, i.e. its cycle matroid is connected. In addition, by Lemma 6 , every graph in $\mathscr{H}_{k}$ is a $u$-fusion of three graphs of the class $\mathscr{H}_{k-1}$. Applying inductively Lemma 5 implies that for any integer $k \geq 0$, all graphs in $\mathscr{H}_{k}$ have $\mu$-pathwidth at least $k+1$. On the other hand, a graph in $\mathscr{H}_{k}$ has clearly $\mu$-pathwidth at most $k+1$, as otherwise its image in $\mathcal{T}_{k}$ over $\phi^{-1}$ would have also linear width more than $k+1$ by Lemma 4 . Summarizing, all graphs in $\mathscr{H}_{k}$ for $k \geq 0$ have $\mu$-pathwidth equal to $k+1$.

Consider now such a graph $H$ in $\mathscr{H}_{k}$ and also the tree $T=\phi^{-1}(H)$. For an edge $e$ in $E(H)=E(T)$, we examine the graph $H / e$ and the tree $T / e$. Since $T$ belongs in $\mathcal{T}_{k}$ all leaves have neighbors of degree 2 and therefore $T / e$ is again a tree with the same number of leaves. Moreover, both $T / e$ and the cycle matroid of $H / e$ are connected. It follows that $H / e=\phi(T / e)$ and hence by Lemma $4, \mu-\mathbf{p w}(H / e) \leq k$, as $T$ is an obstruction for linear width of at most $k$.

Similarly, we examine the graph $H \backslash e$ and the tree $T \backslash e$. Since the cycle matroid $\mathcal{M}(H \backslash e)$ is not connected, it does not hold that $H \backslash e=\phi(T \backslash e)$. However, each connected block $\mathcal{M}_{i}$ in $\mathcal{M}(H \backslash e)$ is the cycle matroid of $\phi\left(T_{i}\right)$, where $T_{i}$ is a minor of $T \backslash e$. In any case, again Lemma 4 immediately implies that $\mu$-pw $(H \backslash e) \leq k$.

Conversely, we will prove that if the dual of a biconnected outerplanar graph is an obstruction to $\mu$-pathwidth then it belongs to the family $\mathscr{H}_{k}$.

Lemma 8. Let $H$ be the dual of a biconnected outerplanar graph that is an obstruction for $\mu$-pathwidth at most $k$. Then $H \in \mathscr{H}_{k}$.

Proof. To a contradiction, suppose that $H$ does not belong to the family $\mathscr{H}_{k}$. Since $H$ is an obstruction for $\mu$-pathwidth at most $k$, surely $\mu$-pw $(H) \geq k+1$ and Lemma 4 implies $\mathbf{l w}(T) \geq k+1$, for the corresponding tree $T=\phi^{-1}(H)$. Thus it contains a minor $T^{\prime} \preceq T$ such that $T^{\prime} \in \mathcal{T}_{k+1}$. Consider now $H^{\prime}=\phi\left(T^{\prime}\right)$ and observe that $H^{\prime} \preceq H$; a contradiction since by definition $H^{\prime} \in \mathscr{H}_{k+1}$.

For every non-negative integer $k$, we define $\mathscr{H}_{k}^{*}$ as the class of all duals of the graphs in $\mathscr{H}_{k}$ (see Fig. 5). The previous two lemmata, along with Lemma 1 , imply the main result of our paper.

Theorem 1. For every non-negative integer $k$, the set $\mathscr{H}_{k}^{*}$ is the obstruction set for the class outerplanar graphs with $\mu$-pathwidth at most $k$.

The theorem reveals a bijection between the acyclic obstructions for linear width and the outerplanar obstructions for $\mu$ pathwidth. This also gives a way to lower bound the size of $\mathcal{P}_{k}$. Copying the counting made in [19] (see also [20]), it follows that $\left|\mathbf{o b s}\left(\mathcal{P}_{k}\right)\right| \geq(k !)^{2}$.

Another consequence of our results is the following.

Corollary 1. Let $G$ be a biconnected outerplanar graph and let $T$ be a tree such that $G^{*}=\phi(T)$. Then $\mu-\mathbf{p w}(G)=\mathbf{l} \mathbf{w}(T)$. 
The latter implies the existence of a linear time algorithm for the computation of the $\mu$-pathwidth of outerplanar graphs.

Corollary 2. There exists a linear algorithm that, given an outerplanar graph, outputs its $\mu$-pathwidth.

Proof. Let $G$ be an outerplanar graph. Let also $G_{1}, \ldots, G_{r}$ be its biconnected components and $H_{1}, \ldots, H_{r}$ their corresponding duals. Let

$$
k=\max \left\{\mathbf{l} \mathbf{w}\left(\phi^{-1}\left(H_{i}\right)\right) \mid i=1, \ldots, r\right\} .
$$

The linear-width of trees can be computed in linear time, using a straightforward adaptation of the linear algorithm of [18] for computing the pathwidth of a graph. Therefore, from Corollary 1 and Lemma 1 we have that $\mu$-pw $(G)=k$.

\section{Acknowledgments}

The first author was supported by the project "Support of Research" of the National and Kapodistrian University of Athens (project code: 10812). The second author was co-financed by the Greek national funds through the Operational Program "Education and Lifelong Learning" of the National Strategic Reference Framework (NSRF) - Research Funding Program: "Thales. Investing in knowledge society through the European Social Fund".

\section{References}

[1] Brian Beavers, James Oxley, Constructive characterizations of 3-connected matroids of path width three, European J. Combin. 29 (7) (2008) $1643-1661$.

[2] Thomas H. Brylawski, A combinatorial model for series-parallel networks, Trans. Amer. Math. Soc. 154 (1971) 1-22.

[3] J.A. Ellis, I.H. Sudborough, J.S. Turner, The vertex separation and search number of a graph, Inf. Comput. 113 (1) (1994) 50-79.

[4] Fedor V. Fomin, Pinar Heggernes, Rodica Mihai, Mixed search number and linear-width of interval and split graphs, Networks 56 (3) (2010) $207-214$.

[5] J.F. Geelen, A.M.H. Gerards, N. Robertson, G.P. Whittle, On the excluded minors for the matroids of branch-width $k$, J. Combin. Theory Ser. B 88 (2) 2003) 261-265.

[6] James F. Geelen, A.M.H. Gerards, Geoff Whittle, Branch-width and well-quasi-ordering in matroids and graphs, J. Combin. Theory Ser. B 84 (2) (2002) 270-290.

[7] Jim Geelen, Bert Gerards, Geoff Whittle, On Rota's conjecture and excluded minors containing large projective geometries, J. Combin. Theory Ser. B 96 (3) (2006) 405-425.

[8] Rhiannon Hall, James Oxley, Charles Semple, The structure of 3-connected matroids of path width three, European J. Combin. 28 (3) (2007) 964-989.

[9] Rhiannon Hall, James Oxley, Charles Semple, Geoff Whittle, Fork-decompositions of matroids, Adv. Appl. Math. 32 (3) (2004) $523-575$.

[10] Illya V. Hicks, Nolan B. McMurray Jr., The branchwidth of graphs and their cycle matroids, J. Combin. Theory Ser. B 97 (5) (2007) 681-692.

[11] Petr Hliněný, The Tutte polynomial for matroids of bounded branch-width, Combin. Probab. Comput. 15 (3) (2006) $397-409$.

[12] Jisu Jeong, O-joung Kwon, Sang-il Oum, Excluded vertex-minors for graphs of linear rank-width at most $k$, in: Natacha Portier, Thomas Wilke (Eds.), 30th International Symposium on Theoretical Aspects of Computer Science, STACS 2013, in: Leibniz International Proceedings in Informatics (LIPIcs), vol. 20, Schloss Dagstuhl-Leibniz-Zentrum fuer Informatik, Dagstuhl, Germany, 2013, pp. 221-232.

[13] Navin Kashyap, Matroid pathwidth and code trellis complexity, SIAM J. Discrete Math. 22 (1) (2008) 256-272.

[14] Daniel Král', Computing representations of matroids of bounded branch-width, in: Wolfgang Thomas, Pascal Weil (Eds.), STACS 2007, in: LNCS, vol. 4393, Springer, Berlin, Heidelberg, 2007, pp. 224-235.

[15] Frédéric Mazoit, Stéphan Thomassé, Branchwidth of graphic matroids, in: Surveys in Combinatorics 2007, in: London Math. Soc. Lecture Note Ser., vol. 346, Cambridge Univ. Press, Cambridge, 2007, pp. 275-286.

[16] James G. Oxley, Matroid Theory, Oxford Science Publications, The Clarendon Press Oxford University Press, New York, 1992.

[17] T.D. Parsons, Pursuit-evasion in a graph, in: Theory and Applications of Graphs: Proceedings Internat. Conf., Western Mich. Univ., Kalamazoo, Mich., 1976, in: Lecture Notes in Math., vol. 642, Springer, Berlin, 1978, pp. 426-441.

[18] Konstantin Skodinis, Construction of linear tree-layouts which are optimal with respect to vertex separation in linear time, J. Algorithms 47 (1) (2003) 40-59.

[19] Atsushi Takahashi, Shuichi Ueno, Yoji Kajitani, Minimal acyclic forbidden minors for the family of graphs with bounded path-width, Discrete Math. $127(1 / 3)(1994) 293-304$

[20] Dimitrios M. Thilikos, Algorithms and obstructions for linear-width and related search parameters, Discrete Appl. Math. 105 (2000) $239-271$.

[21] Robin Thomas, Tree-decompositions of graphs, in: Lecture Notes, School of Mathematics, Georgia Institute of Technology, Atlanta, Georgia 30332, USA, 1996.

[22] W.T. Tutte, Connectivity in matroids, Canad. J. Math. 18 (1966) 1301-1324. 